\title{
Demandas y organización de los estudiantes secundarios: una lectura sociológica más allá de fronteras y análisis coyunturales
}

\author{
Jorge Baeza Correa*
}

\begin{abstract}
Resumen
El presente artículo intenta ingresar a los aspectos más estructurales y transversales de la realidad de los estudiantes secundarios. Avanza más allá del análisis coyuntural de un movimiento estudiantil específico y de un país en particular, da cuenta de cómo América Latina se enfrenta a un proceso de masificación de la educación secundaria, que va asociado a una alta diferenciación interna en cuanto a calidad y con serias dificultades para hacer dialogar la cultura escolar con la cultura juvenil. Se detiene en las nuevas formas que adopta la organización estudiantil para hacer escuchar su palabra, frente a la realidad antes descrita y concluye con algunas reflexiones sobre la importancia del protagonismo de los jóvenes en la educación secundaria.
\end{abstract}

Palabras Clave: Sociología de la Educación / Educación Secundaria / Estudiantes / Movimiento Estudiantil.

\begin{abstract}
The present article attempts to touch onto the most structural and crosssectional aspects of secondary students' reality. It goes beyond the juncture analysis of a specific student movement and of a particular country; it gives an account of how Latin America is faced to a process of massiveness of secondary education, that is associated to a high internal differentiation as to quality and with serious difficulties to connect the school culture with the teenagers' one. The article pays special attention to the new forms that the students' organization adopts to be heard, facing the reality abovementioned and concludes with some reflections on the importance of the youngsters' leading part in secondary education.
\end{abstract}

Key words: Sociology of Education / Secondary Education / Students / Student movement

\footnotetext{
El autor es Sociólogo y Doctor en Ciencias de la Educación, actualmente se desempeña como investigador del Centro de Estudios en Juventud del Departamento de Sociología de la Universidad Católica Silva Henríquez. Para un contacto con el autor, e-mail: jbaeza@ucsh.cl
} 
Los acontecimientos ocurridos en Chile con los estudiantes secundarios, donde miles de ellos, y a lo largo de todo el país, protestaron en las calles y tomaron en su poder los establecimientos escolares, ha dado pie a una enorme cantidad de artículos sobre este accionar, muchos de ellos textos de gran lucidez y enorme calidad $^{1}$. Pero, junto a estos, hay otros muchos, muy concentrados en un análisis de coyuntura donde no se logran identificar las notas distintivas del estadio en que se ubica la realidad de ser hoy alumno o alumna de educación secundaria, y desde la cual se construye el discurso estudiantil; como tampoco ayudan a vislumbrar los nuevos aspectos estructurales que presenta la organización estudiantil, que exigen recurrir a elaboraciones más recientes de la teoría de los movimientos sociales.

En un análisis que supere el dato periodístico (de cientos de entrevistas a sus dirigentes), las cronologías, las declaraciones de adhesión o en contra (muchas disfrazadas de neutrales), e incluso la mirada concentrada solo en un país, sino que tratando de leer sociológicamente las tendencias de la Región, léase América Latina, se hace necesario concentrarse en los dos aspectos ya enunciados: (a) qué significa ser hoy estudiante de educación secundaria en países que están ampliando considerablemente su cobertura educacional y (b) cómo se estructura en la actualidad el movimiento estudiantil, en una sociedad de redes informáticas y de desvalorización de la política de partidos.

\section{Ser hoy estudiantes de educación secundaria}

En la actualidad, un consenso fundamental logrado en el campo de la educación entre los países de América Latina y el Caribe está claramente reflejado en el Proyecto Regional de Educación

1 A modo de ejemplo, ver los excelentes trabajos reunidos en el número especial de la Revista Electrónica Latinoamericana de Estudios sobre Juventud. Año 2 - $\mathrm{N}^{\circ} 3$-1er. Semestre 2006; junio 2006. Número monográfico: Los Estudiantes Secundarios de Chile: cuando los jóvenes se manifiestan... Editada por CELAJU en: www.joveneslac.org/ portal/000/publicaciones/revista/3/index.htm 
de UNESCO": "promover cambios en las políticas educativas a partir de la transformación de los paradigmas educativos vigentes para asegurar aprendizajes de calidad, tendientes al desarrollo humano, para todos a lo largo de la vida" (p. 8). Para el logro de esta finalidad, un principio importante es "que la formulación, ejecución y evaluación de las políticas educativas tengan como centro promover cambios en los diferentes actores involucrados y en las relaciones que se establecen entre ellos" (p. 9). Esto supone -indica el Proyecto Regional- pasar "del lenguaje de los actores al de los autores. El término actor da la idea de ejecutar el papel de un libreto previamente establecido, mientras que autor significa persona que crea, que define su papel y que es causa de un cambio o acción" (p. 9).

En este marco, "los alumnos no son el objeto de la educación sino sujetos de derechos a una educación que potencie al máximo su desarrollo como personas y les permita insertarse e influir en la sociedad en la que están inmersos" (p. 10). Sin embargo, sostiene el Proyecto Regional, "todavía persiste una cultura muy instalada de considerar a los alumnos como meros receptores y reproductores de información y no como sujetos activos en la construcción de conocimientos" (p. 10). "La educación -afirma el Proyecto Regional de UNESCO- ha de tener como centro a los alumnos y considerarlos como protagonistas de su aprendizaje y no como receptores de la enseñanza" (p. 15).

El protagonismo exige la consideración de los alumnos y alumnas en su proceso formativo. No obstante ello, buena parte del ordenamiento de los establecimientos escolares de secundaria, como también de las políticas de este nivel, se construyen ignorando a los jóvenes. De esta forma, el protagonismo de los estudiantes en sus centros escolares queda altamente restringido,

2 UNESCO (2002): Proyecto Regional de Educación para América Latina y el Caribe. Primera reunión intergubernamental del Proyecto Regional, La Habana, Cuba, 14-16 de noviembre 2002. 
llegando incluso a una invisibilización de sus personas y de su cultura juvenil ${ }^{3}$.

La imposibilidad del protagonismo de los jóvenes en sus centros escolares convierte a estos centros -en más de una ocasión- en espacios formativos sordos y descontextualizados: no escuchan a los jóvenes que son sus alumnos ni consideran su realidad histórica y social. Muchas veces se "habla de ellos", de los jóvenes, pero pocas veces se "habla con ellos". Esta falta de diálogo con los y las jóvenes conduce a una visión sobre los alumnos(as) altamente simplificada. Además, se experimenta la interacción pedagógica sin considerar al alumno como un sujeto autónomo, con una identidad propia y situado en un tiempo y un espacio. La realidad de la cultura juvenil no está al margen de los grandes cambios culturales a nivel mundial. Por lo tanto, desatender la cultura juvenil implica formar personas sin consideración de su contexto.

La educación secundaria posee distintas denominaciones y abarca diferentes tiempos entre los países de la Región. Mientras para algunos es una etapa que considera 4 años, para otros es mayor o menor. De aquí la necesidad de asumir, para efectos de comparación internacional, que la educación secundaria tiene relación con la escolaridad post-primaria, que abarca la población que se ubica entre los 13-14 y los 17-18 años ${ }^{4}$ en forma inclusiva.

3 Cfr. Baeza Correa, Jorge (2001): "La visibilidad del joven en la cultura escolar: la transición del rol de estudiante al oficio de alumno(a)". En Revista JOVENes, Revista de Estudios sobre Juventud, editada por Ediciones Nueva Época, Instituto de la Juventud de México. Año $5 \mathrm{~N}^{\circ}$ 14, mayo - agosto; páginas 110-131.

4 Ver, al respecto, Beatriz Macedo y Raquel Katzkowicz (2000): “Educación Secundaria: Balance y Prospectiva". Documento de Discusión para el Seminario sobre Prospectivas de la Educación en la Región de America Latina y el Caribe, organizado por UNESCO, Santiago de Chile, $23 / 25$ de agosto 2000. Se puede ver también, al respecto, el trabajo de Joao Batista Araujo e Oliveira (2000): "Educación Media en América Latina: diversificación y equidad", documento de Apoyo para el mismo Seminario, en la cual se indica que "el término 'nivel secundario' se refiere a aquellos estudios posteriores al nivel primario, cuya duración varía de 3 a 6 años e incluye a los alumnos en los grupos de 13-14 a 16-18 años" (Pág. 4). Definición que es asumida para los efectos del presente texto. 
Hablar, por lo tanto, de educación secundaria es hablar principalmente de jóvenes.

La crisis de la escuela secundaria, colocando en la mirada a los jóvenes como centro, se expresa principalmente en un triple proceso: (a) masificación que genera expectativas y frustraciones que ponen en cuestión su sentido; (b) una alta segmentación interna que da cuenta no solo de calidades distintas sino de vivencias y expectativas muy diferenciadas; $y$, por último, (c) una falta de diálogo entre la cultura escolar y la cultura juvenil, que termina negando el protagonismo a los jóvenes, quienes se suponen el centro del quehacer educativo.

\subsection{Masificación de la Educación Secundaria y cambios culturales en la institución escolar}

Hoy, la educación secundaria, considerando el segmento de edad recién indicado, presenta, como una de sus características más definitorias, la existencia de un proceso de ampliación de cobertura. La tendencia general en los países de América Latina y el Caribe es que el grupo etáreo considerado crece anualmente en escolaridad, con lo cual en muchos países de la región la educación secundaria está dejando de ser un espacio de elite y está en un claro proceso de masificación ${ }^{5}$.

En un reciente trabajo de Claudia Jacinto ${ }^{6}$, queda claro que la mayoría de los países de América latina han subido su asistencia escolar en la población que va entre los 13 y los 19 años. A modo de ejemplo, se indican algunos de los datos señalados por la autora recién citada:

$5 \quad$ El Informe Panorama Educativo de Las Américas, del Proyecto Regional de Indicadores Educativo (resultante de la II Cumbre de Las Américas), indica que la tasa neta de matricula en educación secundaria, en América Latina y el Caribe, alcanzaría al 54\%. Con una dispersión que va de un $20 \%$ en Haití a un 100\% en Barbados.

6 Jacinto, Claudia (2006): La escuela media. Reflexiones sobre la agenda de la inclusión con calidad. E. Fundación Santillana, Buenos aires, Argentina. 
Asistencia Escolar en Áreas Urbanas. Población 13 a 19 años

\begin{tabular}{|l|c|c|}
\hline País & Año & Total \\
\hline \multirow{2}{*}{ Honduras } & 1990 & 57,7 \\
\cline { 2 - 3 } & 2002 & 68,3 \\
\hline \multirow{2}{*}{ Uruguay } & 1990 & 70,6 \\
\cline { 2 - 3 } & 2002 & 76,5 \\
\hline \multirow{2}{*}{ México } & 1992 & 62,7 \\
\hline \multirow{2}{*}{ Chile } & 2004 & 68,0 \\
\hline \multirow{2}{*}{ Costa Rica } & 1990 & 78,6 \\
\hline \multirow{2}{*}{ Brasil } & 2003 & 85,3 \\
\cline { 2 - 3 } & 1990 & 68,6 \\
\hline & 2002 & 76,9 \\
\cline { 2 - 3 } & 1990 & 64,6 \\
\hline
\end{tabular}

Fuente: Elaboración de Claudia Jacinto a base de datos del Panorama Social 2005 de CEPAL

Este proceso de masificación trae consigo desafíos y exigencias en, a lo menos, tres ámbitos: al joven estudiante, al establecimiento escolar y al sistema educativo en general.

\subsubsection{En el alumno}

El proceso de masificación de la Educación Secundaria actual contribuye en lo inmediato a la creación de juventud. Hoy, cada vez más, los integrantes del grupo etáreo que reciben la Educación Secundaria pueden retardar su paso a las responsabilidades de la vida adulta. Se prolonga a través del tiempo una mayor dependencia familiar y un más tardío ingreso al mundo laboral y la salida de la familia de origen.

En la actualidad, los límites demográficos para definir juventud, aquella estratégica salida frente a la imposibilidad de llegar a un acuerdo en el concepto de juventud, están puestos en cuestión. La definición operacional de que joven es aquella persona que tiene entre 15 y 24 años en un muy breve tiempo ha quedado superada; ya muchas investigaciones y estudios extienden la juventud hasta los 29 años (como es el caso de la Encuesta Nacional de Juventud en Chile) y la tendencia parece ir en aumento. La 
edad ha dejado de ser un parámetro fundamental que distingue socialmente una fase de otra y gana cada vez más importancia el factor de "paso o tránsito"7.

La masificación de la escolaridad en definitiva, ha ido generando paulatinamente una fuerte asociación entre educación secundaria y juventud. La vida cotidiana de una población cada vez mayor está estructurada en función de su condición de estudiantes. El mundo intersubjetivo, creado al interior de los establecimientos escolares y los endogrupos que en el se conforman hacen como "naturales" a la vida juvenil la vivencia escolar.

La fácil asociación entre educación secundaria y juventud genera una primera consecuencia social; quien no está integrado al sistema escolar está en falta. El no estar presente en el mecanismo de integración social que es la escuela o liceo se juzga como una desviación al ideal social ${ }^{8}$. Obviamente, esta es una consecuencia de tipo cuantitativo, que simplifica la realidad, donde se parte del dato de que la mayoría es la normalidad y las excepciones, desviaciones a la norma. Pero la masificación de la secundaria no es un proceso donde se avanza en todos los grupos sociales de idéntica forma. Quienes mayoritariamente están fuera del sistema son jóvenes de sectores pobres, de sectores rurales y, preferentemente, indígenas.

7 Ken Roberts argumenta que los problemas y prioridades para la sociología de la juventud, se deben concentrar en la actualidad en las transiciones, ya que estas, en sí mismas, son las inherentes al ser joven y no las edades, que pueden variar y varían significativamente. Cfr. Roberts, Ken (2003) Problems and Priorities for the Sociology of Youth. En: Bennett, Andy; Cieslik, Mark y Miles, Steven (editors) "Researching Youth" Ed. Palgrave Macmilan. Great Britain, 2003, pp. 13-28. Dentro del conjunto amplio de transiciones, en un reciente publicación del Banco Mundial (2006) Informe sobre el desarrollo mundial. El desarrollo y la próxima generación, se sostiene al respecto que "Las decisiones que adopten los jóvenes durante cinco fases de transición -seguir estudiando, comenzar a trabajar, adoptar un estilo de vida saludable, formar una familia y ejercer los derechos cívicos- son las que repercuten en mayor medida, a largo plazo, sobre la manera de dar seguridad al capital humano, desarrollarlo y ponerlo en acción" (Pág. 2).

8 Sapiains Arrué, Rodolfo y Zuleta Pastor, Pablo: "Representaciones sociales de la escuela en jóvenes urbano populares desescolarizados" Revista Última Década $\mathrm{N}^{\circ} 15$, CIDPA, Viña del Mar Chile, Octubre 2001, pp53-72. En: www.cidpa.org/txt/15art_ 02.doc 
Volviendo a la tabla de ejemplificación ya antes presentada, pero complementándola ahora con datos del nivel socioeconómico de los estudiantes, queda expresado claramente lo que se indica:

Asistencia Escolar en Áreas Urbanas Población 13 a 19 años, según quintil de ingreso per cápita del hogar

\begin{tabular}{|l|c|c|c|c|}
\hline \multirow{2}{*}{ País } & Año & Total & $\begin{array}{c}20 \% \text { más } \\
\text { rico }\end{array}$ & $\begin{array}{c}20 \% \text { más } \\
\text { pobre }\end{array}$ \\
\hline \multirow{2}{*}{ Honduras } & 1990 & 57,7 & 79,2 & 51,2 \\
\cline { 2 - 5 } & 2002 & 68,3 & 85,8 & 50,0 \\
\hline \multirow{2}{*}{ Uruguay } & 1990 & 70,6 & 89,4 & 60,5 \\
\cline { 2 - 5 } & 2002 & 76,5 & 94,9 & 64,2 \\
\hline \multirow{2}{*}{ México } & 1992 & 62,7 & 80,7 & 55,6 \\
\cline { 2 - 5 } & 2004 & 68 & 86,2 & 62,2 \\
\hline \multirow{2}{*}{ Chile } & 1990 & 78,6 & 98,6 & 74,3 \\
\hline \multirow{2}{*}{ Costa Rica } & 2003 & 85,3 & 94,1 & 81,4 \\
\hline \multirow{2}{*}{ Brasil } & 1990 & 68,6 & 86,2 & 57,9 \\
\cline { 2 - 5 } & 2002 & 76,9 & 90,2 & 72,9 \\
\cline { 2 - 5 } & 1990 & 64,6 & 86,7 & 56,1 \\
\hline \multirow{2}{*}{ Fuenyyy} & 2003 & 78,4 & 90,5 & 74,5 \\
\hline
\end{tabular}

Fuente Elaboración de Claudia Jacinto a base de datos del Panorama Social 2005 de CEPAL

Se agrega, a lo anterior, que la capacidad del sistema no va a la par en cuanto a integración y posibilidad de retención, situación que trae consigo - unido a lo antes dicho- que la deserción del sistema genera un sentimiento de pérdida, dado el alto reconocimiento de la escuela como el principal lugar para el logro de una identidad juvenil positiva sobre sí mismo y reconocida socialmente.

\subsubsection{En la institución escolar}

La masificación de la educación secundaria constituye, por lo demás, un proceso que es experimentado por la institución escolar, con una lenta respuesta en lo que se refiere a capacidad de modificación para adaptarse a los cambios producidos. Dado que, por años, la educación secundaria fue privativa de una elite y de unos pocos "becarios", se mantiene una imagen canónica del 
alumno que queda desfasada con la llegada de una población de características diferentes.

Todavía en muchos se mantiene una visión de los alumnos de educación secundaria como sujetos sin mayores responsabilidades, lo que es propio de sectores acomodados de la sociedad. De aquí que algunas programaciones escolares no consideren situaciones laborales (o requerimientos propios de algunas estaciones del año en el campo) o de maternidad a temprana edad.

Situación que se complejiza aún más cuando se tienden a extrapolar modos pertinentes para el trabajo con niños a un grupo en edad juvenil. En algunos países, con el aumento de los años de educación primaria obligatoria (de 6 a 8 años de educación básica por ejemplo), se ha tendido a una infantilización de quienes están en los últimos años de básica, cuando en realidad poseen una edad propia del mundo juvenil.

De acuerdo a conocimientos ya acumulados, existen diferencias sustantivas entre Educación Primaria y Educación Secundaria $^{9}$, en la forma en que se experimenta por parte del estudiante. Se reconocen cambios: (a) en las "esferas de justicia"; (b) en la espera de reciprocidad; (c) en la necesidad de desplegar una estrategia escolar y (d) en el desarrollo de una subjetividad no escolar. Estas diferencias muchas veces no son reconocidas ni menos estimuladas para avanzar en autonomía y protagonismo juvenil.

En el paso de Educación Primaria a Educación Secundaria, se asume la existencia de una diversidad en las "esferas de justicia". Mientras el mundo de la infancia (y la escuela básica) está organizado alrededor de una gran "unidad normativa" que rige tanto en el ámbito escolar como en la familia, el mundo de la educación secundaria está regido por la percepción de que existen

9 Coincidiendo con Tenti Fanfani, las particularidades observadas en Francia por Dubet y Martucelli, en cierta medida son válidas en el contexto escolar urbano de América Latina. Tenti Fanfani, Emilio (2000) "Culturas Juveniles y Cultura Escolar". En:http://www.iipe-buenosaires.org.ar/pdfs/seminario_internacional/panel4_ fanfani.pdf 
diversos ámbitos de justicia: una regla se aplica en el recreo, otra entre los amigos y otra dentro de la sala de clases.

Mientras que en la escuela básica el niño tiende a representarse a la autoridad y al maestro como algo natural e indiscutido, el adolescente percibe que el colegio de enseñanza secundaria constituye un mundo complejo donde existe una diversidad de actores con intereses y capacidades diferentes, lo que exige en la relación entre las personas un Principio de Reciprocidad, donde en la relación profesor alumno ya no sea unidireccional (el profesor tiene todo el poder y hace lo que quiere, mientras que el alumno solo tiene que obedecer), sino que es una relación de deberes y derechos recíprocos entre las partes.

Los alumnos y alumnas de Educación Secundaria, a diferencia del niño en la escuela primaria (que percibe que solo basta ser aplicado y obedecer las reglas y a sus superiores para tener éxito), descubren que se requiere del desarrollo de estrategias diversas para enfrentar con éxito la vida escolar. En el colegio o liceo, los adolescentes perciben que "ser alumno" es algo más complejo que seguir ciertos automatismos; por el contrario, el adolescente percibe que para tener éxito es preciso desplegar una estrategia, es decir, que se requiere hacer uso del cálculo, definir objetivos, elegir medios adecuados para los mismos, desplegar la acción en el eje del tiempo, saber esperar, etc. ${ }^{10}$

A diferencia de los niños en las escuelas, que viven una "continuidad relativa" entre su estatuto de niño y su estatuto de alumno, los adolescentes desarrollan una subjetividad no escolar. Se forma una subjetividad y una vida colectiva independientes del colegio o liceo que afecta a la vida escolar misma, e incluso

10 Son estas las razones que permiten hablar con mayor propiedad del "oficio de alumno", como el conjunto de saberes que se logran por apropiación y en forma colectiva en el colegio, y que posibilitan a el alumno o alumna desenvolverse adecuadamente dentro de las "reglas de juego" de su establecimiento (las que no se limitan sólo a la norma escrita e institucional). Ver al respecto Baeza Correa, Jorge $E l$ oficio de ser alumno en jóvenes de liceo de sector popular. Universidad Católica Cardenal Raúl Silva Henríquez, libro serie investigaciones, Nº 19, Santiago, Chile, 2001. 
para algunos adolescentes no resulta fácil lograr articular en forma satisfactoria estos dos espacios de vida, lo que genera, en un mismo sujeto, una tensión entre el estudiante y el adolescente.

\subsubsection{En el sistema}

La masificación de la secundaria suma, a todos los cambios y tensiones anteriores, una realidad de expectativas y frustración para los hijos de las familias que vienen llegando por primera vez. La posibilidad de ingreso a la educación secundaria genera expectativas en los padres que no tienen relación con una realidad de devaluación de la escolaridad. Situación que trae, la mayor parte de las veces y en primer lugar en los jóvenes, una frustración. Los esfuerzos familiares no se ven compensados, como lo fueron en el momento donde solo una minoría alcanzaba la educación secundaria completa.

La devaluación de la escolaridad secundaria, en comparación con los tiempos previos a su masificación implica, por lo demás, una pérdida del sentido y significado social que tenía en sus orígenes. Desde una responsabilidad de formación de las elites (los "herederos", sostienen algunos), se ha convertido en una "obligatoriedad social" (no necesariamente obligatoriedad jurídica) que se hace cada vez más común completarla. Esta pérdida de significado social no es ajena al proceso interno que vive el mismo estudiante que, a diferencia de cuando estaba en la educación primaria, ha desarrollado una capacidad para preguntarse por el sentido y significado de las instituciones en las cuales participa. La respuesta a esta pregunta, instalada en el propio alumno, va a constituir la base de explicación, muchas veces, de las conductas que realiza en su vida escolar.

\subsection{La vivencia de la Educación Secundaria, una realidad altamente diferenciada}

Si bien se conoce y reconoce que el paso de educación primaria a educación secundaria es un tránsito no exento de dificultades, ya 
que implica una primera etapa de "extrañeza" frente a la nueva realidad -dado que se ignoran aspectos básicos del nuevo nivel escolar que se inicia, como a su vez los roles y responsabilidades de las nuevas personas con que se debe interactuar-, no obstante ello, en general, no existen en los establecimientos escolares programas de acogida y menos de inducción.

Esta etapa de "extrañeza", común para todo el que vive la experiencia del paso de primaria a secundaria, se agrava notoriamente cuando ella es acompañada por el cambio de establecimiento escolar, situación que afecta mayoritariamente a los sectores más pobres, ya que las familias de mayor estatus económico mayoritariamente ingresan a centros educativos donde realizan la totalidad de sus estudios (desde lo preescolar hasta el término de la educación secundaria). Quienes experimentan con mayor rigor esta etapa de extrañeza son los alumnos y alumnas de sectores rurales, ya que, en muchas ocasiones, implica el paso obligatorio del campo a la ciudad e incluso del hogar al internado.

Pero, si bien el solo ingreso a la Educación Secundaria ya es motivo de diferencias, una de las características comunes de este nivel de escolaridad en América Latina -junto al ya mencionado proceso de masificación o de clara tendencia hacia a ello- es su notoria segmentación. La diferenciación entre establecimientos, según dependencia (públicos o privados) asociada a niveles de ingreso familiar, es parte de la realidad de la Educación Secundaria actual, y ello se expresa en una diferencia en la calidad de la formación que reciben los estudiantes. Esta segmentación se va haciendo consciente en los alumnos, en la medida que van avanzando en su escolaridad, llegando, muchos de ellos, a visualizar que no es idéntica la formación entre un establecimiento y otro, lo que también sucede entre un estrato social y otro.

La toma de conciencia de las diferencias en calidad conduce a la generación de expectativas diferentes entre los alumnos $\mathrm{y}$, con ello, a una forma de relacionarse con su establecimiento, 
también diferente ${ }^{11}$. Los estudiantes de sectores más pobres, que adquieren conciencia de que la educación secundaria es su etapa última de estudio, no tienen igual forma de relación con los profesores y directivos del establecimiento escolar que quienes ven a esta etapa como un mero paso para el ingreso a la educación superior $\mathrm{y}$, en particular, a la universidad.

Cada grupo social posee un modo particular de experimentar la vivencia de la educación secundaria, donde el futuro se transforma en un organizador del sentido de la educación. De aquí que se reconozcan, a lo menos, tres respuestas diferentes a la pregunta de los alumnos y alumnas de secundaria del para qué ir al colegio o liceo: (a) para un primer grupo, la respuesta es por una obligatoriedad social; (b) para un segundo grupo, la razón obedece a una situación exclusivamente instrumental y, por último, (c) un tercer grupo sostiene que se asiste por el amor al conocimiento. Cada una de estas respuestas, está asociada mayormente a un grupo social en particular. Desde luego que lo último indicado no es mecánico ni excluye que un mismo alumno o alumna exprese una combinación de respuestas.

El asistir a clases "porque sí", porque no hay otra posibilidad, porque es parte de las exigencias de la edad, hace referencia a una primera respuesta ideal-típica: la "obligación como sentido". Esta primera respuesta y modo de vivir la experiencia escolar, está asociada, mayormente, a quienes se ubican en sectores más excluidos, donde no se aprecia un mejor futuro por asistir a clases, pero tampoco existe una alternativa mejor para utilizar el tiempo.

Para algunos alumnos y alumnas, la experiencia escolar es vivida como un tiempo de postergación de beneficios presentes con el fin de beneficios mayores en el futuro, lo que hace de esta respuesta ideal-típica propia de una razón instrumental. Si me

11 Cfr. Kessler, Gabriel (2002): La experiencia escolar fragmentada. Estudiantes y docentes en la escuela media en Buenos Aires. Ed. Instituto Internacional de Planeamiento de la Educación(IIPE)-UNESCOEn:www.crmariocovas.sp.gov.br/pdf/pol/kessler.pdf 
esfuerzo hoy, consiguiré mañana "ser alguien en la vida". Esta respuesta, y con ello este modo de experimentar la educación secundaria, se relaciona con los estratos medios cuyo capital económico, social y cultural, les permite aspirar al ascenso social. Poseen una expectativa de futuro que no tienen los estratos bajos, que le otorga un sentido a la educación de una exigencia del presente para el logro de recompensa en el futuro.

El amor por el conocimiento es la tercera respuesta ideal-típica. El estudiar por el gusto de estudiar, sin una relación interesada, sin un cálculo del beneficio futuro, va a constituir la respuesta, con mayor probabilidad, de los alumnos y alumnas de estratos altos, que están liberados de urgencias y presiones relacionadas con la sobrevivencia. Proyectan, a partir las experiencias familiares previas, un futuro sin mayores sobresaltos, y su amor por el conocimiento les asegura un éxito en la carrera escolar, distinto a quienes viven su presente como un sacrificio en función del futuro.

Esta clara segmentación vertical entre los estratos sociales, se sintetiza en una experiencia escolar muy diferenciada. Los alumnos y alumnas de estratos altos viven con comodidad su experiencia escolar, y tienen confianza en la formación recibida; los estudiantes de estratos medios visualizan una falta de coordinación de esfuerzos, de concordancia entre discurso y práctica, que reconocen que muchos docentes intentan subsanar. Por último, los jóvenes de estratos más bajos viven una experiencia escolar a menudo caótica, de alta permisividad y con poca presión por el estudio.

Frente a esta realidad altamente diferenciada entre estratos, que provoca una clara diferenciación en la calidad de la formación recibida, pero también de la experiencia escolar, la percepción de los alumnos y alumnas también presenta variaciones al momento de enjuiciar la calidad de sus establecimientos educacionales ${ }^{12}$.

12 Villarroel Rosende, Gladis Urenda Salamanca, y Macarena (2001) Los discursos de las alumnas acerca de la escuela: un estudio comparativo En: http:/ / rehue.csociales.uchile. $\mathrm{cl} /$ publicaciones/enfoques/06/GLADYS-VILLARROEL.doc 
Los estudiantes de colegios de estrato más alto utilizan un mayor número de categorías para evaluar su establecimiento que los de estrato más bajo, siendo los primeros, además, más críticos que los segundos. Situación que puede hacer inadecuada la evaluación comparativa de la calidad de los establecimientos, desde el discurso de los alumnos y alumnas, si no se consideran la diferencia en el número de categorías que se utilizan y del significado que se atribuye a cada una de ellas, ya que presentan variaciones para una misma categoría.

Junto a la segmentación vertical ya indicada, de grandes diferencias entre un grupo social y otro, se debe reconocer también una clara diferenciación horizontal al interior de cada estrato, que tiene consecuencia en los alumnos(as). La necesidad de diferenciación de la oferta ha conducido en los estratos altos a una gran heterogeneidad en las pautas de formación y de convivencia escolar; en los estratos medios, las diferencias obedecen a las orientaciones y capacidades de directivos y profesores para generar proyectos educativos y programas de mejora, mientras que, en los estratos bajos, se tiende a estereotipar a los establecimientos como buenos o malos de acuerdo al compromiso que se les atribuye a los docentes, la disciplina de los alumnos, la labor de los directivos y el apoyo de los padres.

Un aspecto que profundiza aún más la desigualdad en la forma de experimentar la Educación Secundaria, aceptada mayoritariamente en la actualidad, es la diferencia e incluso el choque lingüístico que se produce entre el profesor y el alumno(a) cuando provienen de sectores socioculturales diferentes. Los códigos lingüísticos promovidos y difundidos por la cultura escolar resultan muchas veces ajenos y necesarios de aprender para algunos, principalmente para los sectores más pobres, que recién vienen llegando como grupo social a la Educación Secundaria.

El etnocentrismo y la autorreferencialidad del profesor, que se incorpora explícita o implícitamente en la relación pedagógi- 
ca hasta convertirse en parte fundamental de la cultura escolar, imponen de forma invisible una exigencia que deja en desventaja a quienes no tienen incorporados los códigos lingüísticos que él maneja, ni son parte de su práctica cotidiana ${ }^{13}$. Esta situación, que afecta gravemente en la Región a las minorías étnicas (asociadas a sectores rurales e indígenas), muchas veces es disminuida en los diagnósticos educacionales, en lo que se refiere a la realidad de los estratos bajos urbanos que no son necesariamente étnicamente diferenciados ${ }^{14}$.

Una de las dificultades mayores de los alumnos(as) de los estratos bajos urbanos, que ayuda a perjudicarlos en su rendimiento escolar, es la falta de reconocimiento y aceptación de su lenguaje (que es calificado de deficiente e incorrecta) por parte de quienes dirigen los procesos formativos, lo que los deja carentes de una lengua legítima y con ello de una identidad cultural reconocida. Deslegitimación, por lo demás, agravando esta realidad, que no es visible -en muchos casos- para quienes la practican, ya que queda en el "punto ciego" de su autorreferencialidad. Situación, en definitiva, que hace de la vivencia escolar de los alumnos y alumnas de estratos bajos urbanos una experiencia diferente a los de otros estratos.

Por último, una clara expresión de las diferencias de cómo se vive la educación secundaria entre los diversos estratos sociales tiene relación con la forma de expresión del malestar de los alumnos y alumnas en sus establecimientos educacionales ${ }^{15}$.

13 Cfr. Gómez, Luis Fernando (2001): "Privilegio reconocimiento y evaluación de lenguaje: una mirada a los códigos sociolingüísticos en la cultura escolar". Tonos Digital, Revista Electrónica de Estudios Filológicos. № 2, noviembre 2001, Colombia. En: www.um.es/tonosdigital/znum2/estudios/LuisFGomeztonos2.htm

14 Cfr. Mandujano Bustamante, Fernando (2002) "El salvaje en el aula: los problemas de lenguaje e identidad en el rendimiento de estudiantes pobres urbanos". Revista Sepiensa, Chile.

En:www.sepiensa.cl/listas_articulos/articulos_sepiensa/2002/02_febrero_ 2002/20020221_fram.html

15 IIPE-UNESCO (2001) Violencia en Ámbitos educativos. Informes Periodísticos Publicación $N^{o}$ 1, julio 2001, Argentina. En: http://www.iipe-buenosaires.org.ar/ prensa/informes/pdfs/informe01_violencia.pdf 
En las clases más altas, son más comunes las agresiones verbales y la exigencia de los alumnos a los adultos, todo ello sin mayor cuestionamiento a la norma establecida. En los estratos medios, se repiten las agresiones verbales y las molestias son dirigidas a la institución, en este caso las normas institucionales son cuestionadas. En el caso de los estratos bajos, hay agresiones no solo verbales sino que también físicas, e incluso a las personas (no solo a la propiedad); sin embargo, las normas se acatan, sin mayor discusión, sometiéndose a la autoridad.

\subsection{La distancia entre la cultura escolar y la cultura juvenil}

Si bien, como se ha indicado, la edad de cursar la educación secundaria es la propia de la edad juvenil, buena parte del ordenamiento de los establecimientos escolares de secundaria se construye ignorando a los jóvenes. De esta forma, la cultura escolar no solo no integra a la cultura juvenil, sino que -muchas veces- le cierra las puertas y le da su espalda.

En muchos centros escolares, se experimenta la interacción pedagógica sin considerar al alumno como un sujeto autónomo, situado en un tiempo y un espacio que, si bien no lo determina, lo condiciona poderosamente. Paradójicamente, esta no consideración del contexto se revierte totalmente al momento de explicar, por parte de los educadores, las causas de los bajos niveles de logros de sus alumnos, donde la principal causa de explicación es la propia responsabilidad del estudiante (o des-responsabilidad), acompañada por la falta de apoyo familiar y de su contexto social.

La falta de diálogo con los alumnos(as) conduce a una visión, sobre ellos, altamente simplificada que se expresa en una homogenización de los estudiantes, donde desaparece la persona y se ve restrictivamente como alumno; una etiquetación que hace distinciones entre alumnos a partir de estereotipos y de una 
desconfianza en ellos, ya que se cree que el joven solo actuará correctamente si es vigilado.

Hoy se reconoce el valor de la diversidad, pero aún no se acepta con facilidad la pluralidad de los jóvenes (seguramente -y con razón- por el número de alumnos por docente y el número de horas de docencia directa), y se les sigue tratando como una masa uniforme de alumnos en que cada uno de ellos carece de una identidad propia.

Esta situación, que conduce a una falta de profundización en la identidad de cada joven, se ve complementada -agravando la situación- por la alta valoración que se le otorga al conocimiento escolar (los contenidos impartidos en las actividades curriculares), por sobre el conocimiento de sí mismo por parte del alumno. El desarrollo afectivo de los alumnos(as) no constituye en la mayoría de los casos un tema de preocupación central, o de una valoración de igual medida que los contenidos de las diversas disciplinas que conforman el plan de estudios de educación secundaria.

Temas como el embarazo y la paternidad adolescente, por ejemplo, que constituyen parte de la realidad cultural actual, si bien hoy son recepcionados por los establecimientos escolares, aún son tratados como un problema social, pero sin lograr interpelar la visión del alumnado. En general, los centros educacionales miran al embarazo y a la paternidad temprana como algo no deseado, casi como un accidente, sin considerar que, para más de una joven o joven actual, alumna(o) de educación secundaria, el embarazo o paternidad puede ser algo buscado (son la generación de mayor información sobre métodos de control de natalidad e, incluso, no faltan quienes construyen su proyecto de vida a partir de la maternidad / paternidad ${ }^{16}$ ). La institución escolar sigue

16 Cfr. IIPE-UNESCO (2003) ¿Alumnos o madres y padres adolescentes? Informes Periodísticos Publicación 17, agosto. En: http://www.iipe-buenosaires.org.ar/ prensa/informes/pdfs/informe17_madresadolesc.pdf 
mirando al alumno(a) como un niño que requiere protección y no asume los cambios que hoy son parte de la cultura juvenil.

Acciones de construcción cultural, como la producción musical de numerosos jóvenes, siguen siendo, para la cultura escolar, conductas extramuros, desaprovechando el potencial educativo que ellas poseen ${ }^{17}$. En la juventud actual, el producto cultural de mayor consumo es la música y, en torno a ella, se crean grupos musicales de diversos estilos que les permiten insertarse en circuitos culturales alternativos más amplios. La producción musical permite que los jóvenes pasen desde espectadores pasivos a creadores activos (muchas veces en contextos que les niegan la posibilidad de la creación); posibilita el ejercicio de decidir y comprometerse por lo que se ha escogido, lo que alimenta la responsabilidad; proporciona -al adherir a un determinado estilouna forma alternativa de vivir su condición juvenil, que educa en la forma de relacionarse con lo demás y facilita la construcción de identidad. Cada una de estas potencialidades es ignorada por la cultura escolar por la falta de diálogo con la cultura juvenil.

La disciplina sigue siendo el elemento central que media entre la cultura escolar y la cultura juvenil. El elemento ordenador de la convivencia escolar -en la mayoría de los establecimientoses la disciplina, la que se expresa en una práctica unidireccional de un claro fundamento de carácter adultocéntrico, situación que no tiene tanta relación con los adultos en sí mismos, sino con la matriz en que ésta práctica se ubica, que asume un estilo de relación asimétrica (que incluso en algunos casos se da entre alumnos). No es un problema de edad, sino de actitudes, discursos y estilos que van configurado y dando vida a una particular forma de relación donde prima la verticalidad.

No obstante lo anterior, no se puede dejar de reconocer que, cada vez más, se aprecia la necesidad de los profesores y direc-

17 Cfr Juarez Dayrell: “Juventude, Produçao Cultural e Ecola” En: www.siape.com.br/ seemg/textos/dayrellj001.rtf 
tivos de construir una legitimidad frente a los alumnos, La unidireccionalidad ya no basta en una sociedad de mayor reconocimiento de los derechos de las personas. Los alumnos se saben sujetos de derechos y se hacen respetar. No se puede dejar de reconocer, a este respecto, que entre los fenómenos de las últimas décadas se encuentra la irrupción de la infancia y la juventud como un actor social protagónico, lo que genera nuevas demandas y reflexiones sobre su lugar en la sociedad, la cultura y la educación. La educación deja de ser una práctica de transmisión de valores y de códigos de las viejas generaciones, para avanzar a una modalidad de mayor autonomía y protagonismo de los alumnos y alumnas.

Hoy las relaciones intergeneracionales presentes en los establecimientos de educación secundaria se debaten entre tres vértices de una tríada que es una misma realidad ${ }^{18}$ : (a) la de un mundo adulto que victimiza al mundo juvenil, donde los jóvenes son invisibilizados, al ser tratados solo como alumnos, sin reconocimiento de las singularidades personales y donde el poder de decisión está absolutamente en manos de los adultos; (b) en un segundo vértice, la de un mundo adulto que se debilita frente al mundo juvenil que se posiciona (cabe aquí la difícil relación entre el adulto docente y el joven alumno, donde este último maneja un cúmulo de información lograda de Internet mayor que la del primero), $y$, finalmente, (c) en un tercer vértice, la presencia de los miedos adultos ante un mundo juvenil que rechaza las posturas adultocéntricas, que cuestionan las relaciones de poder hasta ahora existentes y que busca cambiar la asimetría. Todas y cada una de estas posibilidades están presentes e influyen en la relación entre generaciones que implica la interacción pedagógica profesor / directivo - alumno / alumna.

18 Duarte, Claudio: “Mundos jóvenes, mundos adultos: la generación y reconstrucción de los puentes rotos en el Liceo. Una mirada desde la convivencia escolar". En: www. mineduc.cl/media/lpt/zonas/doc/liceos/mundos_jovenes.doc 


\section{Las nuevas formas de la organización estudiantil para hacer valer su opinión}

Toda la realidad antes descrita es la base para que el discurso de los estudiantes se eleve en más de una ocasión como malestar y trascienda a la opinión pública. En esas ocasiones, las formas de organización del movimiento estudiantil dan cuenta, también, de significativos cambios con relación a las décadas pasadas.

El movimiento de los estudiantes secundarios en las recientes manifestaciones realizadas en Chile ha llamado poderosamente la atención a algunos y a otros los ha descolocado, por su forma de estructuración interna, donde los dirigentes son reemplazados por voceros y las decisiones no se adoptan en las cúpulas sino en asambleas.

Estudios actuales sobre los movimientos sociales resaltan a lo menos cinco características de las nuevas formas de organización ${ }^{19}$ :

- Valores. Rechazan las premisas de una sociedad basada en el crecimiento económico y buscan una nueva relación con la naturaleza, el propio cuerpo, el sexo, el trabajo, el consumo y se enfocan a un estilo de vida de realización de la individualidad.

- Formas de acción y organización. Formas no convencionales de acción antagónicas a la política tradicional; organización en pequeña escala, descentralizada, anti-jerárquica que favorece la democracia directa.

- Constitución. Reclutan principalmente aquellos que pagan los costos del proceso de modernización y aquellos más sensibles a los problemas resultantes de dicho proceso.

19 Ver al respecto a Aranda Sánchez, María José (2000): “El Movimiento Estudiantil y la Teoría de los Movimientos Sociales". En Revista Convergencia, enero-abril, año 7, número 21. Universidad Autónoma del Estado de México; Toluca, México; pp. 225250. En: http://redalyc.uaemex.mx 
- Nuevas aspiraciones. Tránsito hacia valores post-materiales, como un ambiente agradable y buena educación.

- Satisfacción de necesidades puestas en peligro. Producto de los cambios en las relaciones sociales y laborales, pérdida de la identidad y declinación de las relaciones tradicionales ligadas a lealtades.

Dentro de este conjunto de características, una de las que más se destaca por su expresión organizativa es la democracia directa; entendida básicamente como una forma de democracia donde los ciudadanos participan directamente en el proceso de toma de decisiones.

Si bien la democracia directa posee una larga historia, dado que se remonta a la antigua democracia ateniense, con el paso del tiempo se pierde y se reemplaza por un sistema de representantes elegidos por los ciudadanos (lo que se conoce como democracia representativa). Hoy, en el mundo actual, muchos movimientos sociales buscan la evolución desde el actual sistema de democracia representativa vigente, hacia algún tipo de democracia más directa o deliberativa.

En tiempos modernos, la aparición de la informática hace pensar a muchos en volver a la idea de una democracia directa. Por una parte, la disconformidad del ciudadano promedio con el accionar de sus representantes y, por otra, la disponibilidad actual de medios telemáticos que vuelven a hacer posible el ideal de que todos los ciudadanos expresen su voluntad, han llevado a una revalorización de la democracia directa.

Quienes revindican la democracia directa se oponen a la democracia representativa, cuestionándole que se basa en la elección de "representantes" que, una vez elegidos, se arrogan el derecho de "representar" sin ningún mecanismo que asegure el control de estos "dirigentes" por parte de las bases que los eligieron. 
Las críticas a la democracia directa, a su vez, se concentran en cuestionar su practicidad y eficiencia, decidir mediante asambleas -se indica- que es lento y costoso, lo que provoca fatiga y luego apatía (se caería en un "asambleísmo estéril"). Otra desventaja es que las asambleas pueden desembocar en demagogia, se termina escuchando a los que más hablan o tienen más facilidades para expresar sus puntos de vista. Por último, se cuestiona también la dificultad para tratar, mediante este medio, temas complejos (resulta más fácil para preguntas cortas y respuestas dicotómicas, lo que puede generar la elección de políticas incoherentes.

La respuesta a estas críticas va por las posibilidades del Internet como medio para recoger la opinión y que, en las democracias representativas, nada asegura que se puedan escuchar todas las voces (priman los profesionales de la política) y que el problema de decisiones inconsistentes también está presente en esa fórmula.

Hoy, cuando una de las características principales de las culturas juveniles presentes es ser parte de una generación video-formada, que nace entre pantallas y redes ${ }^{20}$; en palabras de Castells, que son habitantes de la sociedad red, los movimientos sociales y -entre ellos- en forma muy especial el movimiento estudiantil, encuentran en Internet un poderoso instrumento que les facilita su acción y organización. "Pero, ¿qué es lo específico?, se pregunta Castells ${ }^{21}$; ¿qué le confiere especificidad a la movilización social a partir del hecho de que se haga por Internet? Bien, hay tres rasgos que son fundamentales en la interacción entre Internet y los movimientos sociales. El primero es que asistimos, en la sociedad, fuera de Internet, a una crisis de las organizacio-

20 Ver al respecto: Feixa, Carles Generació@ La joventut al Segle XXI. Ed. Observatori Català de la Joventut, Aportacions 12, Barcelona, 2002. También el trabajo de De la Villa Moral Jiménez, María y Ovejero Bernal, Anastasio (2005) "Iuventus digitalis y Iuventus ludens como tipologías psicosociológicas de jóven@s contemporáne@s” En Intervención Psicosocial, 2005, vol. 14 n. ${ }^{\circ} 2$ págs. 161-175.

21 Castells, Manuel “Internet y la sociedad red". En: http:/ / www.uoc.edu/web/esp/ articles/castells/print.html 
nes tradicionales estructuradas, consolidadas, tipo partidos, tipo asociaciones de orientación directamente política, y, además, se produce la emergencia de actores sociales, fundamentalmente a partir de coaliciones específicas sobre objetivos concretos (...). Es decir, en general, en la sociedad hay un salto de los movimientos sociales organizados a los movimientos sociales en red a base de coaliciones que se constituyen en torno a valores y proyectos. Internet es la estructura organizativa y el instrumento de comunicación que permite la flexibilidad y la temporalidad de la movilización, pero manteniendo, al mismo tiempo, un carácter de coordinación y una capacidad de enfoque de esa movilización".

El segundo rasgo, sostiene Castells, es que los movimientos sociales se desarrollan, cada vez más, en torno a códigos culturales, a valores, "son movimientos que dependen sobre todo de la capacidad de comunicación y de la capacidad de llevar a cabo un reclutamiento de apoyos y de estímulos mediante esa llamada a los valores, a los principios y a las ideas (...). La transmisión instantánea (a través de Internet) de ideas en un marco muy amplio permite la coalición y la agregación en torno a valores"22. Internet permite, además, desvelar con facilidad aquellos movimientos sociales que no se constituyen en torno a lo que dicen que son. "El tercer rasgo específico de los movimientos sociales es que, cada vez más, indica Castells, el poder funciona en redes globales y la gente tiene su vivencia y construye sus valores, sus trincheras de resistencia y de alternativa en sociedades locales. (...) Internet permite la articulación de los proyectos alternativos locales mediante protestas globales que acaban aterrizando en algún lugar, pero que se constituyen, se organizan y se desarrollan a partir de la conexión Internet, es decir, conexión global, de movimientos locales y de vivencias locales. Internet es la conexión global-local, que es la nueva forma de control y de movilización social en nuestra sociedad" ${ }^{23}$.

22 Ídem.

23 Ídem. 


\subsection{Nuevas formas de organizarse para hacer politica}

Al margen del debate teórico de las fortalezas y debilidades de una democracia directa en la sociedad actual, un dato irrefutable es que distintos Movimientos Sociales en general, y en forma muy principal en el Movimiento Estudiantil -los principios que dan vida a la democracia directa- se han ido instalado paulatinamente y, al parecer, gozan de buena salud y poseen una esperanza de vida que augura que se debe aprender a conocer, respetar y valorar esta modalidad de gestión.

Las organizaciones generadas o que asumen la lógica de la democracia directa poseen una estructura donde la participación de todos los integrantes de la organización es facilitada por su horizontalidad, la relevancia de la base, la consideración de espacios micro (naturales a las personas como son sus propios cursos, lo que facilita además la discusión de sus problemas más cercanos) y la existencia de mecanismos de control para el aseguramiento del respeto a las decisiones tomadas.

Una organización representativa de esta modalidad, a modo de ejemplo, es el CGR (Consejo General de Representantes), organización que representa a los estudiantes de la Facultad de Filosofía y Humanidades de la Universidad de Chile, el cual se presenta a sí mismo como una "experiencia alentadora para todo el movimiento estudiantil Chileno, secundario o universitario". El CGR "se estructura a partir de las bases de cada curso, establece una serie de mecanismos de control de parte de los estudiantes a la hora de las decisiones que se tomen y apunta a dejar el poder de esas decisiones en sus manos. El sistema es el siguiente: cada curso y carrera de la Facultad discute en una asamblea los problemas locales o generales del movimiento estudiantil. Luego, las propuestas, las decisiones y votaciones que cada curso efectúe son expresadas por sus voceros, que son totalmente revocables, y que deben llevar la voz de la asamblea a cada instancia superior de decisión, a las asambleas de Facultad (al CGR) o, por ejemplo, a la FECH (Federación de Estudiantes de la Universidad de 
Chile). Las decisiones expresan, así, la voluntad y los intereses del conjunto de los estudiantes. Además, cada estudiante puede asistir a las reuniones de Carrera o al CGR de Facultad para dar sus opiniones y para poder 'controlar' que los delegados expresen fielmente las decisiones, las posiciones y el voto que representan" 24 .

Situación muy similar a la Asamblea Coordinadora de Estudiantes Secundarios [ACES] en Chile, que está claramente consignada en el relato de su origen. "Lo primero fue establecer lo innecesario que es una organización estudiantil sin tejido social que la impulse. Por ende, construir desde arriba, con los cerebros dirigenciales trabajando a mil por hora, escribiendo declaraciones fue desechado. No es la ACES una 'coordinadora de presidentes de CCAA', sino una de estudiantes organizados, lo que resalta la importancia de construir movimiento en micro en los liceos, como base de lo que se establezca arriba. Solo una organización real en las bases posibilitará que, en algún momento, se vuelva a hablar de federación representativa, y la construcción de ese tejido fundamental será tarea de todos quienes trabajen en la ACES (...). La autonomía es un principio rector, así como la horizontalidad, que debe entenderse como 'acción directa de masas', lo contrario a delegar esa acción en un dirigente. Por esto, no se plantea jerarquización ni dirección central como necesidad. Cada sector que se integre tiene la misma importancia. Las decisiones se toman abajo, en asambleas de libre convocatoria. Las clásicas directivas y sus funciones y atribuciones son reemplazadas por comisiones ejecutivas que se ajustan a las resoluciones por todos tomadas y las ejecutan públicamente"25.

24 "Una organización de democracia directa estudiantil. La experiencia del CGR". Clase contra Clase $\mathrm{N}^{\mathrm{o}}$ 34, 05-04-2002 por: Miguel L En: http://www.clasecontraclase. $\mathrm{cl} /$ periodico.php?nota $=134$

25 Comisión Congreso 2001, Documento de Discusión: Historia ACES. En: www. nodo50.org/aces/documentos/documento_feses.htm 


\subsection{Las características comunes de las organizaciones de democracia directa}

Numerando los cambios que trae consigo esta estructuración distinta a la de carácter tradicional de las organizacionales tanto políticas como sociales, se puede indicar lo siguiente:

- Inversión de la lógica piramidal tradicional, donde el vértice superior constituye el punto mayor de energía. En las organizaciones de estructura tradicional, es en la cúspide donde descansa habitualmente (no necesariamente reconocido en el discurso explícito) la tarea de proponer, motivar, conducir e incluso evaluar; situaciones todas que justifican el calificativo de "dirigente", para quienes dirigen. En las organizaciones con democracia directa, los dirigentes son reemplazados por "voceros", que dan a conocer la voz de sus representados. Su tarea no es dirigir, sino ser portador de la voz de la asamblea.

- A diferencia también de los dirigentes, los voceros son "voceros revocables", es decir sobre ello está siempre la posibilidad de su rápido cambio. Ello, a diferencia de la tarea dirigencial, donde el dirigente es elegido por un período por lo general no menor de tiempo, y donde el revocar su mandato no es una trámite de fácil factura.

- La posibilidad de un alto y rápido control sobre los voceros ayuda, además, a una fuerte disciplina de respeto de los acuerdos; entre ellos, la mantención del silencio cuando así fue decidido.

- Los voceros, como uno entre sus pares, cumplen una tarea más dentro de la organización, la que, en su interior, articula un complejo tejido que posibilita, a sus integrantes, participar en numerosas áreas y comisiones de trabajo. Situación que aporta al involucramiento e identificación con la organización. 
- La valoración de la igualdad genera, al interior de las organizaciones, un cuidado por la presencia femenina en las tareas de representación. Se cuida que, tanto en las labores de vocería como en la distribución de tareas diversas, exista equilibrio en términos de género.

- La red interna de trabajo de las organizaciones con democracia directa hace una utilización permanente de los medios tecnológicos para la planificación y discusión sobre sus acciones, las que, una vez ejecutadas -utilizando nuevamente los medios tecnológicos- son evaluadas y difundidas. Para este fin, los teléfonos celulares, el internet, el chat, los blog, las fotos digitales, etc., se utilizan con gran masividad y versatilidad.

- El aprendizaje e incorporación del valor de la comunicación hacen también que estas organizaciones sean diestras en el mensaje hacia los otros, ya no solo en la comunicación interna. Frases cortas y fotos impactantes son parte de la agenda de comunicación diaria hacia quienes se quiere llegar, incluidos los medios de comunicación de masas.

\subsection{El movimiento estudiantil y su relación con los movimientos políticos}

Las organizaciones estudiantiles que asumen la lógica de una democracia directa, por lo general, no se inscriben ni adhieren a un partido político en particular, sino que mantienen una autonomía de las orgánicas partidarias. Conducta que es, por lo demás, acorde a su discurso de respeto de las diferencias e igualdad de las personas. Ello no implica que quienes integran estas organizaciones no posean posiciones político-partidistas, sino que sus posiciones son un elemento más en el diálogo interno, en la democracia de la organización.

Lo anterior tampoco implica que estas organizaciones no tengan opciones políticas. Lo común es que las organizaciones 
de democracia directa posean opciones políticas claras, pero su discurso sobre el particular diferencia la política de lo político. Lo primero, lo valoran y relevan como inherente a la vida social, incluso cuestionan a quienes no adoptan una posición política o tratan de pasar como apolíticos. Lo segundo, como organización (como un todo mayor a la suma de sus individualidades), muchas veces rechazan lo político como un vicio de las democracias representativas, que termina convirtiéndose en un espacio ajeno y lejano, e incluso profesionalizado, que gira sobre sí mismo alejándose de la vida y necesidades de las personas.

Un aspecto importante a considerar en este ámbito es que, al interior de las organizaciones que asumen esta forma de estructuración, conviven las tradicionales orgánicas partidarias con otras y nuevas formas de agrupación juvenil. De esta forma, desde colectivos culturales hasta adhesiones musicales y/o a equipos de fútbol se articulan y crean complejos vínculos en función del interés que los llevó a organizarse.

No obstante la autonomía y horizontalidad indicadas, no se puede dejar de reconocer lo indicado por José María Aranda Sánchez $(2000)^{26}$ respecto de que los movimientos estudiantiles se componen de una masa mayoritaria de jóvenes que participan esporádicamente y un grupo de "activistas que continuamente están llevando a cabo acciones diversas que, de alguna manera, mantienen la actividad del movimiento" (p. 243). El núcleo activista -indica Aranda- desempeña las siguientes funciones: “a) conservación y actualización de la memoria colectiva del movimiento; b) actitud vigilante ante los acontecimientos, manteniendo siempre algunas pre-demandas del movimiento; c) inquietar a las masas estudiantiles sobre los problemas y las acciones a tomar, y movilización de las mismas; d) núcleo organizador del movimiento; e) planteamiento de las demandas; f) formular el discurso de la protesta estudiantil; y g) centro del mantenimiento material e ideológico del movimiento" (p. 243).

26 Aranda Sánchez, José María: Op. Cit. Pág. 243. 


\section{Conclusión: La importancia del protagonismo de los jóvenes en la escuela secundaria}

Ser joven, en la realidad actual, tiene profundas diferencias con las sociedades pasadas donde existían líneas que señalaban exactamente el momento de transición de la juventud a la edad adulta. "En las sociedades arcaicas, afirma José Machado Pais (2000), los rituales de la circuncisión. Más tarde, el matrimonio y el inicio del trabajo eran momentos clave para adquirir la condición de adulto. Y se decía del servicio militar que hacía 'hombres' a los muchachos (y, en tiempo de guerra, además, acababa con ellos). Actualmente, las líneas que señalan las fronteras entre la juventud y la edad adulta son más vagas, y los jóvenes cada vez más consideran la vida como algo inestable, fluctuante, discontinuo y reversible" (p. 91).

La realidad de límites difusos se convierte, en la juventud, en un dato con el cual se convive sin mayor dificultad, pero no solo en su expresión externa, como podrían ser los espacios de hibridez cultural, sino que en las propias vidas juveniles, donde en un mismo joven se manifiestan, en más de una ocasión, conductas que dan cuenta -principalmente a los ojos de los mayores- de una verdadera "fragmentación". En palabras de Jesús Martín Barbero (2002), “...quizá ninguna otra figura como la del flujo televisivo para asomarnos a las rupturas y las formas de enganche que presenta la nueva experiencia cultural de los jóvenes. La programación televisiva se halla fuertemente marcada, a la vez, por la discontinuidad que introduce la permanente fragmentación -cuyos modelos en términos estéticos y de rentabilidad se hallan en el video-clip publicitario y el musical- y por la fluida mezcolanza que posibilita el zapping, el control remoto al televidente, especialmente al televidente joven ante la frecuente mirada molesta del adulto, para armar 'su programa' con fragmentos o 'restos' de deportes, noticieros, concursos, conciertos o películas" (p. 4). 
Esta realidad de trayectorias no lineales y fragmentación va haciendo de la juventud una experiencia distinta de la vivida por el mundo adulto. Algo propio y diferente pero que, a su vez, en su interior, también se diferencia no existiendo una sino diversas culturas juveniles. En palabras de Carles Feixa (1998), “en un sentido amplio, las culturas juveniles se refieren a la manera en que las experiencias sociales de los jóvenes son expresadas colectivamente mediante la construcción de estilos de vida distintivos, localizados en el tiempo libre o en espacios intersticiales de la vida institucional" (p. 84). Expresiones heterogéneas entre sí que no están exentas de influencias del medio pero que permiten diferenciar a los jóvenes de los adultos y a los jóvenes entre sí.

$\mathrm{Al}$ interior de cada una de estas microsociedades, diversas manifestaciones simbólicas son reordenadas y recontextualizadas, logrando como resultado la construcción de un estilo juvenil propio que se manifiesta, principalmente, en un lenguaje como forma de expresión oral distintiva de los adultos, la adopción de una música que, por su consumo y creación, marca una identidad grupal y, por último, una estética que identifica visualmente al grupo (forma de llevar el pelo, ropa o accesorios). El joven de hoy -como en otras épocas- busca decir, con su lenguaje, música y estética, quién es él, a qué grupo adhiere.

No se puede dejar de reconocer que estas conductas poseen, además de la búsqueda de una identidad propia, mucho de cierta resistencia social frente a la realidad de un mundo globalizado que uniforma las conductas de las personas; de una sociedad con Estados que ceden su espacio a las leyes del mercado y ponen en riesgo las identidades nacionales (que muchas veces han sido constituidas desde el propio Estado); de un derrumbe, además, de las utopías e ideologías que entregaban un mega-relato para explicar todos los campos de la vida.

La apropiación de ciertos espacios como propios, verdaderos territorios juveniles, caracterizados por concentrar bares, plazas 
y canchas de fútbol, como también la acción del graffiti -donde los jóvenes marcan sus territorios con los nombres de los grupos con que se identifican, ya sea musicales o futbolísticos, pero también con sus propias "chapas" (apodos)- son, en definitiva, afirmación de identidad, de manifestación de sentimientos personales o propios de los grupos de pertenencia.

Con una finalidad similar (demostrar identidad) es posible ver también la apropiación del propio cuerpo y la inscripción de su propio sello distintivo en el uso de perforaciones corporales (piercings) y tatuajes (tatoo), lo que nos habla en definitiva de verdaderas tribus urbanas que, haciendo uso del espacio urbano-corporal con una gran diversidad de sentidos y significados, construyen identidad en un mundo que obstaculiza su surgimiento ${ }^{27}$. Estas nuevas manifestaciones culturales juveniles, de una verdadera tribalización como expresión de subjetividad, nacen, además, por la búsqueda de afectos, de nuevos tipos de relaciones que dejen de lado las construcciones marcadas por la racionalidad. Es una vuelta a lo tribal, a lo afectivo-emocional, propio de la comunidad, compensando la atomización y la disgregación de las grandes urbes.

A pesar de la enorme diversidad que actualmente caracteriza a la cultura juvenil, ella, sostiene Juan Carlos Tedesco (2003) ${ }^{28}$, "registra algunos elementos comunes que son contrarios, antagónicos, o al menos muy diferentes de los que privilegia la cultura escolar. La cultura juvenil otorga mucha importancia al cuerpo, a la música, a las formas personalizadas de la religión, al predominio de la imagen, a la empatía con las nuevas tecnologías de comunicación, a la afectividad como dimensión de la personalidad

27 Es importante indicar que estas "resistencias", estas formas de diferenciación y construcción de identidad son muy bien aprovechadas por las grandes cadenas comerciales, que ponen a la venta los accesorios que dan identidad (la música con la cual se protesta, entre otros productos).

28 Juan Carlos Tedesco: "Problemas y tendencias de reforma en América Latina". En Germán Rama (editor): Alternativas de Reforma de la Educación Secundaria. Banco Interamericano de Desarrollo, 2003. 
y de las relaciones sociales, y al presente como dimensión temporal fundamental. Esta nueva concepción cultural implica, de algún modo, una pérdida de la importancia de ciertos elementos centrales y clásicos de la cultura escolar, como son el predominio de la lectura, la valorización del conocimiento y del trabajo sistemático, la postergación de satisfacciones, la valoración del pasado como patrimonio a transmitir, y del futuro, como proyecto para el cual es preciso formarse".

Una educación de calidad exige reconocer a actores y autores implicados. Exige, en el caso de los estudiantes, escuchar su $\mathrm{voz}^{29}$, que se articula desde sentimientos y vivencias, que no necesariamente se expresa en los códigos acostumbrados del mundo adulto ${ }^{30}$. Es un lenguaje propio que, para escucharlo, previamente hay que reconocerlo. En las escuelas y liceos, no solo hay alumnos(as); hay jóvenes, ciudadanos y ciudadanas, con una serie de derechos y deberes. Su valor no radica en la potencialidad de ser ciudadanos del futuro; son ciudadanos y ciudadanas ahora. De aquí la necesidad de conocer y abrir espacio, en la cultura escolar, a la presencia de la cultura juvenil, una cultura vigorosa y a momentos ambigua, viva e impertinente, que muchas veces se deja solo transitar por los márgenes, quedando como una "allegada" en la casa que también le pertenece. Sin un conocimiento sociológico de los alumnos y alumnas de hoy, es difícil el logro de una educación de calidad.

29 Baeza Correa, Jorge (2002): "Leer desde los alumnos(as), condición necesaria para una convivencia escolar democrática". En: UNESCO: Educación Secundaria: un camino para el Desarrollo Humano. Santiago de Chile. En Internet en: http: / / www.cidpa.org/ txt/articulos/Unescoart\%EDculo.doc

30 Conocer cómo hoy se estructuran las organizaciones juveniles resulta una necesidad fundamental para el diálogo con ella. Respetar su forma de organización, por lo tanto sus tiempos y sus modalidades de toma de decisión, de seguro implica también un cambio para quienes interactúan con ella, ya que les obliga a salir desde sus lógicas aprendidas y asumidas en sus estructuras organizativas tradicionales. 


\section{Bibliografía consultada}

Araujo e Oliveira, Joao Batista (2000). Educación Media en América Latina: diversificación y equidad. Documento de Apoyo para el Seminario sobre Prospectivas de la Educación en la Región de América Latina y el Caribe, organizado por UNESCO, Santiago de Chile, $23 / 25$ de agosto 2000 .

Aranda Sánchez, María José (2000). "El Movimiento Estudiantil y la Teoría de los Movimientos Sociales". En Revista Convergencia, eneroabril, año 7, número 21. Universidad Autónoma del Estado de México; Toluca, México; pp. 225-250. En: http://redalyc.uaemex.mx

Baeza Correa, Jorge (2001). "La visibilidad del joven en la cultura escolar: la transición del rol de estudiante al oficio de alumno(a)". En Revista JOVENes, Revista de Estudios sobre Juventud Editada por Ediciones Nueva Época, Instituto de la Juventud de México. Año $5 \mathrm{~N}^{\circ}$ 14, mayo - agosto; páginas 110-131.

(2001). El oficio de ser alumno en jóvenes de liceo de sector popular. Universidad Católica Cardenal Raúl Silva Henríquez, libro serie investigaciones, $\mathrm{N}^{\mathrm{o}} 19$, Santiago, Chile.

(2002). "Leer desde los alumnos(as), condición necesaria para una convivencia escolar democrática". En: UNESCO: Educación Secundaria:uncaminoparaelDesarrolloHumano.SantiagodeChile.En: http:/ /www.cidpa.org/txt/articulos/Unescoart\%EDculo.doc

Banco Mundial (2006). Informe sobre el desarrollo mundial. El desarrollo y la próxima generación. En: http://siteresources.worldbank.org/ INTWDR2007/Resources/1489782-1158107976655/overviewes.pdf

Castells, Manuel. Internet y la sociedad red. En: http:/ / www.uoc.edu/web/ esp/articles/castells/print.html

Comisión Congreso (2001). Documento de Discusión: Historia ACES. En: www.nodo50.org/aces/documentos/documento_feses.htm

De la Villa Moral Jiménez, María y Ovejero Bernal, Anastasio (2005). “Iuventus digitalis y Iuventus ludens como tipologías psicosociológicas de jóven@s contemporáne@s" En Intervención Psicosocial, 2005, Vol. 14 n. ${ }^{\circ} 2$ págs. 161-175.

Duarte, Claudio. "Mundos jóvenes, mundos adultos: la generación y reconstrucción de los puentes rotos en el Liceo. Una mirada desde la convivencia escolar". En: www.mineduc.cl/media/lpt/ zonas/doc/liceos/mundos_jovenes.doc 
Feixa, Carles. Generació@La joventut al Segle XXI. Ed. Observatori Català de la Joventut, Aportacions 12, Barcelona, 2002.

Gómez, Luis Fernando (2001). "Privilegio reconocimiento y evaluación de lenguaje: una mirada a los códigos sociolingüísticos en la cultura escolar". Tonos Digital, Revista Electrónica de Estudios Filológicos. No 2, noviembre 2001, Colombia. En: www.um.es/tonosdigital/znum2/estudios/LuisFGomeztonos2.htm

IIPE-UNESCO (2001). “Violencia en Ámbitos educativos". Informes Periodísticos Publicación No 1, julio 2001, Argentina. En: http:// www.iipe-buenosaires.org.ar/prensa/informes/pdfs/informe01_violencia.pdf

IIPE-UNESCO (2003). “¿Alumnos o madres y padres adolescentes?” Informes Periodísticos Publicación 17, agosto. En: http:/ /www. iipe-buenosaires.org.ar/prensa/informes/pdfs/informe17_ madresadolesc.pdf

Jacinto, Claudia (2006). La escuela media. Reflexiones sobre la agenda de la inclusión con calidad. E. Fundación Santillana, Buenos aires, Argentina.

Juárez, Dayrell. "Juventude, Produçao Cultural e Ecola" En: www.siape. com.br/seemg/textos/dayrellj001.rtf

Kessler, Gabriel (2002). "La experiencia escolar fragmentada. Estudiantes y docentes en la escuela media en Buenos Aires". Ed. Instituto Internacional de Planeamiento de la Educación (IIPE) - UNESCO En: www.crmariocovas.sp.gov.br/pdf/pol/kessler.pdf

La experiencia delCGR. “Clase contra Clase”. No 34, 05-04-2002 por Miguel L. En: http:/ / www.clasecontraclase.cl/periodico.php?nota=134

Macedo, Beatriz y Katzkowicz, Raquel (2000). Educación Secundaria: Balance y Prospectiva. Documento de Discusión para el Seminario sobre Prospectivas de la Educación en la Región de América Latina y el Caribe, organizado por UNESCO, Santiago de Chile, 23/25 de agosto 2000.

Mandujano Bustamante, Fernando (2002). "El salvaje en el aula: los problemas de lenguaje e identidad en el rendimiento de estudiantes pobres urbanos". Revista Sepiensa, Chile. En: www.sepiensa. cl/listas_articulos/articulos_sepiensa/2002/02_febrero_ 2002/20020221_fram.html

Proyecto Regional de Indicadores Educativos. Informe Panorama Educativo de Las Américas. Editado por el Proyecto Regional de Indicadores Educativos resultante de la II Cumbre de Las Américas. 
Revista Electrónica Latinoamericana de Estudios sobre Juventud. Año 2 - $\mathrm{N}^{\circ} 3$ -1er. Semestre 2006; junio 2006. Número monográfico: Los Estudiantes Secundarios de Chile: cuando los jóvenes se manifiestan... Editada por CELAJU. En: www.joveneslac.org/portal/000/ publicaciones/revista/3/index.htm

Roberts, Ken (2003). "Problems and Priorities for the Sociology of Youth". En: Bennett, Andy; Cieslik, Mark y Miles, Steven (editors). Researching Youth. Ed. Palgrave Macmilan. Great Britain, 2003.

Sapiains Arrué, Rodolfo y Zuleta Pastor, Pablo. Representaciones sociales de la escuela en jóvenes urbano populares desescolarizados. Revista Última Década. $\mathrm{N}^{\circ} 15$, CIDPA Viña del Mar Chile, Octubre 2001, pp. 53-72. En: www.cidpa.org/txt/15art_02.doc

Tedesco, Juan Carlos. "Problemas y tendencias de reforma en América Latina". En Germán Rama (editor). Alternativas de Reforma de la Educación Secundaria. Banco Interamericano de Desarrollo, 2003.

Tenti Fanfani, Emilio (2000). "Culturas Juveniles y Cultura Escolar". En: http://www.iipe-buenosaires.org.ar/pdfs/seminario_internacional/panel4_fanfani.pdf

UNESCO (2002). Proyecto Regional de Educación para América Latina y el Caribe. Primera reunión intergubernamental del Proyecto Regional, La Habana, Cuba, 14-16 de noviembre 2002.

Villarroel Rosende, Gladys; Urenda Salamanca, Mariana Macarena (2001). Los discursos de las alumnas acerca de la escuela: un estudio comparativo. En: http://rehue.csociales.uchile.cl/publicaciones/enfoques/06/GLADYS-VILLARROEL.doc 